\title{
Uso de estrategias de afrontamiento de los pacientes con el VIH y el cáncer en una clínica privada en Santa Marta - Colombia
}

\author{
Use of coping strategies of patients suffering from HIV and cancer \\ at a public clinic in Santa Marta, Colombia
}

\section{Guillermo Augusto Ceballos Ospino', Amparo Echeverri Arias², María Paola Jiménez Villamizar²}

\begin{abstract}
Resumen
En esta investigación se identifica y analiza el uso de estrategias de afrontamiento en pacientes con VIH y cáncer dentro de una clínica privada de la ciudad de Santa Marta, Colombia. Objetivo: Identificar y analizar las estrategias para hacer frente a la enfermedad en pacientes con VIH y cáncer ingresados en una clínica privada en la ciudad de Santa Marta, Colombia, para mejorar el proceso de adaptación a la enfermedad, logrando modificar esquemas y comportamientos en relación a su salud, además de promover conductas saludables y actitudes de autocuidado en este tipo de pacientes. Material y métodos: Se realizó un estudio transversal, con una muestra de carácter aleatorio simple de 30 pacientes en total, de los cuales 22 eran portadores del VIH y 8 eran pacientes con Cáncer. Resultados: Los pacientes con VIH al igual que los de cáncer, no cuentan con la capacidad para afrontar la situación adversa que presenta.
\end{abstract}

Palabras clave: VIH, Cáncer, adaptación a la enfermedad, adherencia al tratamiento.

\begin{abstract}
Objective: To identify and analyze strategies to cope with the disease in patients suffering from HIV and cancer admitted to a private clinic in the city of Santa Martha, Colombia, in order to improve the adaptation process to the disease, modifying schemes and behaviors in relation to their health; as well as to promote healthy habits and self-care attitudes in this kind of patients. Materials and Methods: It was carried out a cross-sectional study with a
\end{abstract}

simple random sample of 30 patients from which 22 were HIV carriers and 8 were cancer patients. Results: Patients with HIV as well as those with cancer are not capable of coping with this adverse situation.

Keywords: HIV, cancer, adaptation to the disease, adherence to the treatment.

\section{Introducción}

Entre las enfermedades que generan mayor temor y preocupación a nivel global, se encuentran el VIH-SIDA y Cáncer, y aún más por el desarrollo epidemiológico que ha presentado en los últimos años debido a los altos índices de morbilidad y mortalidad, requiriendo así una atención inmediata.

En lo que concierne a los cambios de tipo físico y biológico que se encuentran en relación al proceso salud-enfermedad para el afrontamiento del VIH-SIDA y el cáncer son diversos, pero tienen una cierta relación en los aspectos psicológicos, comportamentales y emocionales debido a la adaptación que se mantiene por dichos diagnósticos, estos aspectos inician las sospechas si se padece VIHSIDA o Cáncer, empezando a generar un malestar subjetivo a quienes lo padecen. El proceso de adaptación y aceptación de estas enfermedades son dos de los factores que causan mayor disfuncionalidad en el paciente, en especial la infección por VIHSIDA donde se demanda una práctica consistente y eficiente de los comportamientos de adhesión al tratamiento antirretroviral en sí y otras como el apoyo al mismo, aunque en estudios recientes se han reconocido otras variables psicológicas y sociales (depresión, estrés, bajos niveles de motivación y carencia de apoyo social) que entorpecen la práctica de esos comportamientos, favoreciendo así el progreso clínico de la enfermedad y una reducción en la calidad y tiempo de vida en esta población ${ }^{[1]}$.

1. Psicólogo especialista - Universidad del Magdalena

2. Estudiantes de grado Universidad del Magdalena

Datos de contacto: Guillermo Augusto Ceballos Ospino, correo electrónico: guillermoceballos@gmail.com/gceballos@unimagdalena.edu.co Recibido: 07/06/2014 - Revisado: 08/06/2014 - Aceptado: 30/06/2014 
La infección por VIH es una enfermedad que se identifica por una disminución progresiva de los linfocitos T-CD4, considerados como un subgrupo de células encargadas de regular la respuesta inmune. Esta enfermedad persiste por un tiempo sin ningún tipo de síntomas, en esta etapa inicial la persona se le diagnostica con el virus de inmunodeficiencia humana (VIH). En la segunda etapa donde aparecen los síntomas el virus en el organismo supera la capacidad del sistema inmune, haciendo a la persona más vulnerable a infecciones oportunistas, en esta fase la persona ha llegado a la etapa de SIDA. ${ }^{[2,3]}$

La psicología se consolida cada día en el área de la salud ${ }^{[4-6]}$, En diversos estudios se ha encontrado que factores como el estrés, los estados emocionales y la manera de afrontar una situación influyen en el curso de enfermedades crónicas como es el caso del VIHSIDA. En lo que corresponde a trastornos como la depresión y el estrés producen cambios en el sistema inmunológico, endocrino y nervioso, de que una u otra manera juegan un papel importante en la manifestación y curso de la enfermedad ${ }^{[7-9]}$.

Las personas infectadas con VIH-SIDA suelen presentar sentimientos de vergüenza, baja autoestima, culpa, castigo y en situaciones extremas ideaciones suicidas. En lo que corresponde en sus hábitos sociales, laborales, educativos, salud o familiares manifiestan conductas de aislamiento, este actuar y sentir está basado en un recelo a ser blanco de insultos, amenazas verbales o físicas, acompañadas del miedo de no poder entablar una relación de pareja. En lo que corresponde al enfrentamiento de la enfermedad como es el VIH-SIDA influyen muchos factores tales como la forma en que fue comunicado el diagnóstico, el apoyo social, las percepciones y creencias sobre la enfermedad, la información que tenga de esta misma ${ }^{[10]}$.

El VIH-SIDA es interpretado por los pacientes como una amenaza en varias esferas de sus vidas, tales como: integridad, autonomía, independencia, bienestar, relaciones de familia, amigos, pareja, planes futuros y bienestar económico ${ }^{[11]}$. Por lo cual se ha visto importante el estudio dirigido a identificar estilos de afrontamiento que pudieran tener relación con alteraciones y el progreso de la infección por $\mathrm{VIH}^{[12]}$

El rol del psicólogo en lo que compete a la intervención en personas diagnosticadas con VIH-SIDA, está orientado hacia un proceso interno con el propósito de un cambio psicológico. Desde una vista de la prevención tiene como objetivo reducir daños existentes, buscando favorecer la resolución de situaciones por las que personas atraviesan y evitando mayor vulnerabilidad ${ }^{[10]}$.

Por su parte, el Cáncer se ha convertido en una palabra temida para los que no lo tienen e impronunciable para los que lo padecen. Esta enfermedad de característica maligna y a su vez de delicado pronostico, es la resulta de una proceso evolutivo que sigue una cadena de fases basadas en la existencia de un periodo entre la manifestación de un carcinógeno y el desarrollo de una neoplasia. Dicho proceso, que se produce a causa de cualquier tipo de célula que se puede reproducir incontroladamente desde los diversos tejidos del organismo, no es una enfermedad única, sino un contiguo de enfermedades con sintomatología y curso clínico muy variado en función del lugar y órgano que afecte la célula de origen ${ }^{[13]}$.

Se ha estimado que los pacientes con Cáncer son sujetos sin más cambios psíquicos que la población general, y que se reaccionan ante el estrés que presume la situación de su enfermedad y los agentes concernientes a ésta. Así, las respuestas al estrés pueden estar relacionadas con tipos de personalidad que se exhiben en mayor o menor grado en cada persona ${ }^{[14,15]}$. Según Oblitas ${ }^{[16]}$, las respuestas afectivas ante el hecho de presentar un diagnóstico de Cáncer, están mediadas por gran variedad de rasgos y estilos de afrontamiento interrelacionados con diversas clases de personalidad. Por ejemplo, el hecho de que un paciente con cáncer evidencie características de personalidad tipo $\mathrm{C}$, que se identifica por el retraimiento y privación de reacciones afectivas de carácter negativa, tales como la ansiedad, agresividad e ira, lo que a su vez logra trascender en la manera como dicho paciente confronta su enfermedad ${ }^{[17]}$.

Las respuestas frente a la experiencia de padecer Cáncer son reacciones individuales y de características subjetivas, tanto ante el diagnóstico como ante el transcurso del tratamiento (respuestas que fluctúan desde la confusión, la aprensión, el pánico, la desconcierto, la rabia, la desesperanza, etc.) y por lo tanto, aquellos pacientes crónicos percibirán, consciente o inconscientemente, su vida bajo peligro inminente, provocada por el quebranto de salud y la resultante frustración y dolor que simboliza el estar en inmediación con su vulnerabilidad física-corporal ${ }^{[18]}$.

Considerando que concurren determinados asomos acerca que la expresión de emociones como la ira, la cólera y la presentación de actitudes de espíritu de lucha y de ganas de vivir, han favorecido en el proceso de afrontamiento del Cáncer y que, en caso inverso, los individuos que admiten su enfermedad y ostentan comportamientos de conformismo y de contención de emociones, manifestar explosiones afectivas, han precipitado el proceso neoplásico y han impedido su recuperación, respalda la idea de que los pacientes que declaran rasgos predominantes de la personalidad tipo C, asumirán más conflictos en su proceso de afrontamiento de la enfermedad ${ }^{[16]}$.

Cabe matizar el rol protagónico de las entidades gubernamentales que orientan la salud pública en cuanto a la renovación de los mecanismos psicoeducativos, orientados hacia la prevención, que permitan optimizar los hábitos saludables, propiciar mayor calidad de vida y prevenir enfermedades, es por esto que se evaluó el uso de estrategias de afrontamiento, buscando a su vez implementar a largo plazo un programa de entrenamiento en el uso de las mismas, haciendo énfasis en las referidas al entorno, que están enfocadas a transformar las presiones del entorno, las barreras, los recursos y los procedimientos y las referidas al sujeto, encargadas del cambio motivacional o cognitivos, como la transición de los niveles de aspiraciones, la disminución de la asistencia del yo, la exploración de conductos diferentes de gratificación, el desarrollo de nuevos modelos de comportamiento o el aprendizaje de recursos y procedimientos nuevos ${ }^{[19]}$. Existen numerosas estrategias que potencian el uso de recursos de afrontamiento en un individuo. El automatismo de unas $\mathrm{u}$ otras en buena medida suele estar establecido por la naturaleza del estresor y las ocurrencias en las que se produce. Por ejemplo, las situaciones en las que se puede hacer algo constructivo favorecen estrategias focalizadas en la solución del problema, mientras que las situaciones en las que lo único que cabe es la aceptación favorecen el uso de estrategias focalizadas en las emociones ${ }^{[20]}$. El afrontamiento es entonces un proceso en el que se hacen esfuerzos constantes para manejar el estrés psicológico generado por una situación ${ }^{[21]}$. Estas circunstancias son controladas en una forma particular dependiendo de las estrategias que se utilizan cuando de alguna manera se está acostumbrado a ellas. Sin embargo hay ocasiones en que las condiciones del ambiente representan una amenaza para la integridad del ser y es en este momento en el que la persona actúa para evitar que estos estresores superen su capacidad para afrontarlos y puedan disminuir los recursos disponibles para hacerlo $^{[22]}$

Las disyuntivas psicológicas del abordaje que el profesional en psicología debe efectuar a través del proceso terapéutico en torno al uso de estrategias de afrontamiento, pueden considerarse el 
mantener la calidad de vida del paciente, graduar el nivel de impacto psicológico de los eventos por los que el paciente está atravesando, optimizar el control de la situación por medio de diferentes instrumentos y medios contextualizadas a su entorno y situación ${ }^{[23]}$, todo esto, teniendo en cuenta las características individuales de cada sujeto evaluado, la fase de la enfermedad en las que se encuentren y en cuanto al Cáncer, la diversidad diagnostica que esta afección a nivel biológico presenta.

Es por esto que el objetivo del presente estudio identificar y analizar en los pacientes con VIH y CA el uso adecuado de estrategias y recursos de afrontamiento que le permitan un mejor proceso de adaptación a la enfermedad logrando modificar esquemas y comportamientos en relación a su salud.

\section{Materiales y métodos}

La población fue de 120 pacientes, de la cual por muestreo aleatorio simple se trabajó con una muestra constituida por 30 pacientes, 15 hombres y 15 mujeres, de rango de edades entre 20 y 80 años, diagnosticados con VIH-SIDA y Cáncer; de los cuales 22 pacientes pertenecen al primer grupo y 8 pacientes presentan un cuadro clínico de Cáncer, de una Clínica privada de la ciudad de Santa Marta durante entre Marzo y Junio de 2014

Como criterios de inclusión, se encuentra el cuadro clínico o diagnóstico, donde solo hacían participe de este estudio pacientes cuya enfermedad fuera Cáncer o VIH/SIDA. A su vez se tuvo en cuenta la edad de los evaluados, para que pudieran tener la capacidad intelectual de dar su consentimiento y aprobación en la realización de dicha investigación, tomando como referencia también que el CSI se aplica principalmente a población joven/adulta.

A su vez, dentro de los criterios de exclusión se encuentra la tasa de mortalidad, aquellos pacientes que en el transcurso de la ejecución del estudio fallecían, al no lograr completar todo el proceso, se excluía su participación dentro de la investigación.

Cabe hacer mención, que el número de pacientes con VIH es mayor a la muestra participativa de los de Cáncer, debido que en el periodo que se realizó dicho estudio, la tasa de ingreso a la clínica de los pacientes con VIH era mayor que los del otro grupo. Así mismo, en la población inicial, la tasa de mortalidad de los pacientes con Cáncer fue más representativa en relación a los pacientes con VIH.

A pesar de que la presente investigación no presenta riesgos para la salud, se tuvo en cuenta lo estipulado en la Resolución 008430 de 1993, por la cual se establecen las normas científicas, técnicas y administrativas para la investigación en salud. Así mismo, lo establecido en la ley 1090 de 2006, en especial el apartado de bioética, teniéndose en cuenta el Artículo 50. Donde los profesionales de la psicología al planear o llevar a cabo investigaciones científicas, deberán basarse en principios éticos de respeto y dignidad, lo mismo que salvaguardar el bienestar y los derechos de los participantes.

\section{Instrumentos}

Para evaluar las estrategias de afrontamiento que usaban los pacientes participantes del programa, se aplicó: El Inventario de estrate-

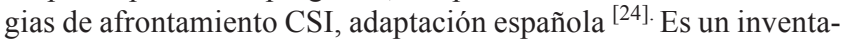
rio autoevaluativo, diseñado para evaluar el uso de estrategias de afrontamiento, este consta de 40 ítems que mide el grado de utilización de estrategias de afrontamiento, diferenciando entre estrategias de afrontamiento activo o dirigidas a la acción (resolución de problemas, reestructuración cognitiva, expresión de emociones y apo- yo social), y estrategias de afrontamiento pasivo o no dirigidas a la acción (evitación de problemas, pensamientos ansiosos, autocrítica y retirada social), con un formato de respuesta tipo Likert de cinco intervalos (desde "nunca utilizada" a "siempre utilizada").Este instrumento maneja la alfa de Cronbach entre .70 y .86, siendo el promedio de .8125

\section{Procedimiento}

Inicialmente se procedió a seleccionar los pacientes que participarían en la identificación y análisis del uso de estrategias de afrontamiento, esto se realizó a través de un muestreo aleatorio simple. Fueron evaluados a través de la escala CSI Inventario de estrategias de afrontamiento, adaptación española, para evaluar estrategias tales como la resolución de problemas, autocritica, expresión emocional, pensamiento desiderativo, apoyo social, reestructuración cognitiva, evitación de problema y retirada social. Los instrumentos fueron aplicados de forma auto administrada por alguno de los autores según las instrucciones propias de los procedimientos psicométricos de evaluación de la personalidad (confidencialidad de la información, inexistencia de respuestas correctas, espontaneidad de respuesta, etc.) haciéndose especial hincapié en las instrucciones específicas del CSI.

Todos los datos fueron procesados con el programa estadístico SPSSvis 18 .

\section{Resultados}

De acuerdo al trabajo realizado, a través de la escala de evaluación de estrategias de afrontamiento, estos fueron los resultados más relevantes encontrados, cabe resaltar la importancia del uso de estas debido al proceso del manejo de situaciones de estrés, en este caso el ser diagnosticado y padecer enfermedades crónicas como el VIH/ SIDA y el Cáncer.

Se logró determinar que de los 30 pacientes evaluados 22 están diagnosticados con VIH-SIDA y 8 con Cáncer. En cuanto al sexo masculino se encontró que 12 fueron diagnosticados con VIH-SIDA y 3 con cáncer, en cuanto a el sexo femenino 10 padecen de padecen de VIH-SIDA y las 5 faltantes Cáncer (Tabla 1).

\section{Tabla 1. Diagnóstico y sexo de la muestra}

\begin{tabular}{lccc} 
Diagnóstico & $\begin{array}{c}\text { Femenino } \\
\%(n)\end{array}$ & $\begin{array}{c}\text { Masculino } \\
\%(n)\end{array}$ & $\begin{array}{c}\text { Total } \\
\%(n)\end{array}$ \\
& & & \\
VIH/ SIDA & $45,5(10)$ & $54,4(12)$ & $100(22)$ \\
Cáncer & $62,5(5)$ & $37,5(3)$ & $100(n)$ \\
\hline
\end{tabular}

De acuerdo al rango de edades, se evidencia que los pacientes con VIH que superan los 60 años presentan mejores estrategias de afrontamiento a la enfermedad, que aquellos que se encuentran en edades entre los 30 a 50 años. En proporción a los pacientes con cáncer, no hay relación alguna entre la edad y el uso de estrategias de afrontamiento (Tabla 10).

Teniendo en cuenta las ocho estrategias de afrontamiento que evalúa la escala, los resultados agrupados en tales estrategias, quedaron así: 
En la Tabla 2 se observa el uso de la estrategia de afrontamiento "resolución de problemas" donde de los 30 pacientes valorados, el $100 \%$ de dicha población no logra un adecuado manejo en el intento de solucionar directamente la situación, ya sea mediante acciones directas, agresivas, o potencialmente arriesgadas.

En el uso de la estrategia "autocritica" se evidencia que todos los pacientes con Cáncer manejan una autocrítica al igual que los diagnosticados con VIH-SIDA, a excepción de un solo paciente, esto se traduce a que en un $97 \%$ reconocen el papel que hayan tenido en el origen o mantenimiento del problema, a bajo o mayor escala (Tabla 3 ).

En la estrategia "expresión emocional" lo que corresponde a los pacientes con VIH-SIDA, no hay mayor distinción entre respuesta, registrándose que 13 de los 22 evaluados si manifiestan expresión emocional frente a los 9 que valoraron la respuesta "no". Es decir que rigen esfuerzos para controlar los propios sentimientos y respuestas emocionales. En cuanto a los pacientes con Cáncer, sobresale la misma anterior respuesta, registrándose un número de 6 pacientes frente a los 8 participantes (Tabla 4).

La estrategia "pensamiento desiderativo" se evidencia que, 11 de los pacientes evaluados no presentan el uso de un pensamiento irreal improductivo, mientras que 19 si manifiestan ideas tipo «Ojalá hubiese desaparecido esta situación». No se registra mayor contraste entre género y diagnóstico (Tabla 5).

En la Tabla 6 el uso de la estrategia "apoyo social" se refleja que 23 de los 30 pacientes evaluados, no presentan apoyo social, es decir que no muestran una red de soporte que les sirva de ayuda,

Tabla 2. Uso de la Estrategia de Afrontamiento Resolución de problemas

\begin{tabular}{|c|c|c|c|}
\hline \multicolumn{4}{|c|}{ Resolución de Problemas } \\
\hline & & $\mathrm{Si}$ & No \\
\hline & & $\%(n)$ & $\%(n)$ \\
\hline \multirow[t]{2}{*}{ VIH/SIDA } & ○ & - & $50(11)$ \\
\hline & q & - & $50(11)$ \\
\hline \multirow[t]{2}{*}{ CANCER } & ○ & - & $37,5(3)$ \\
\hline & Q & - & $62,5(5)$ \\
\hline
\end{tabular}

Tabla 3. Uso de la Estrategia de Afrontamiento Autocritica

\begin{tabular}{|c|c|c|c|}
\hline \multicolumn{4}{|c|}{ Autocrítica } \\
\hline & & $\mathrm{Si}$ & No \\
\hline & & $\%(n)$ & $\%(n)$ \\
\hline \multirow[t]{2}{*}{ VIH/SIDA } & б & $54,5(12)$ & - \\
\hline & q & 40,9 (9) & $4,5(1)$ \\
\hline \multirow[t]{2}{*}{ CANCER } & ð & $37,5(3)$ & - \\
\hline & ㅇ & $62,5(5)$ & - \\
\hline
\end{tabular}

Tabla 4. Uso de la Estrategia de Afrontamiento Expresión Emocional.

\begin{tabular}{|c|c|c|c|}
\hline \multicolumn{4}{|c|}{ Expresión Emocional } \\
\hline & & $\mathrm{Si}$ & No \\
\hline & & $\%(n)$ & $\%(n)$ \\
\hline \multirow[t]{2}{*}{ VIH/SIDA } & ๙ & $36,3(8)$ & $18,1(4)$ \\
\hline & q & $22,8(5)$ & $22,8(5)$ \\
\hline \multirow[t]{2}{*}{ CANCER } & $\sigma^{7}$ & $12,5(1)$ & $25(2)$ \\
\hline & $q$ & $12,5(1)$ & $50(4)$ \\
\hline
\end{tabular}

Tabla 5. Uso de la Estrategia de Afrontamiento Pensamiento Desiderativo

\begin{tabular}{|c|c|c|c|}
\hline \multicolumn{4}{|c|}{ Pensamiento Desiderativo } \\
\hline & & $\mathrm{Si}$ & No \\
\hline & & $\%(n)$ & $\%(n)$ \\
\hline \multirow[t]{2}{*}{ VIH/SIDA } & ð & $36,4(8)$ & $18,2(4)$ \\
\hline & $q$ & $31,8(7)$ & $13,6(3)$ \\
\hline \multirow[t]{2}{*}{ CANCER } & $\sigma^{\pi}$ & $25(2)$ & 12,5 (1) \\
\hline & $q$ & $25(2)$ & $37,5(3)$ \\
\hline
\end{tabular}

Tabla 6. Uso de la Estrategia de Afrontamiento Apoyo Social. Apoyo Social

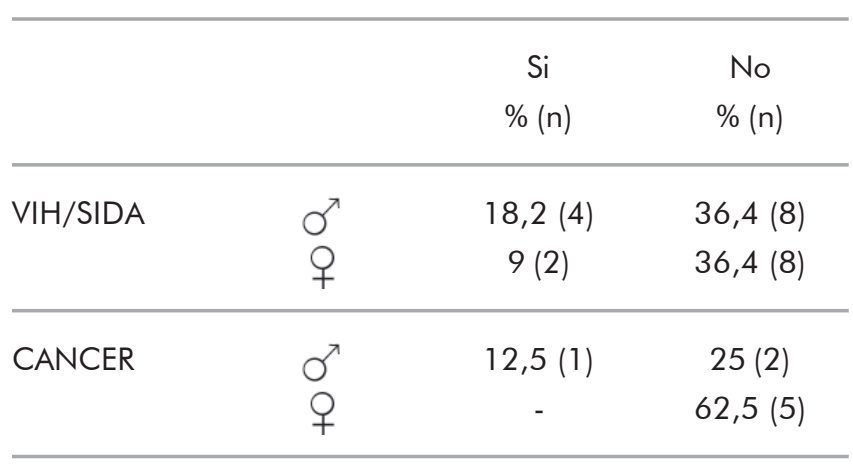

información o también comprensión y apoyo emocional. Se denota que todas las mujeres con Cáncer exhiben dicha situación, manifestando igualdad en número en los diagnosticados con VIH-SIDA, frente a la misma respuesta.

En la utilización de la estrategia "reestructuración cognitiva" el género femenino, en su gran mayoría, 14 de 15, no hacen uso de esta estrategia de afrontamiento, es decir que no logran percibir los 
posibles aspectos positivos que tenga o haya tenido la situación estresante, representando entonces una pequeña muestra de 7 pacientes que si utilizan dicha ayuda como ficha de afrontamiento (Tabla 7).

La estrategia "evitación de problemas" se obtuvo una mayor proporción en la respuesta "si", donde el $97 \%$ de los pacientes si presentan un aparente intento de apartarse del problema, no pensar en él, o evitar que le afecte. No se observa mayor contraste entre géneros o diagnóstico (Tabla 8).

En la Tabla 9 el uso de la estrategia "retirada social" se evidencia también una preferencia en la respuesta "si", donde al igual que en la anterior tabla, el $97 \%$ de los evaluados evitan el contacto con otros, o manifiestan un aislamiento y represión del componente emotivo que les genera su situación.

Tabla 7. Uso de la Estrategia de Afrontamiento Reestructuración Cognitiva

\begin{tabular}{|c|c|c|c|}
\hline \multicolumn{4}{|c|}{ Reestructuración cognitiva } \\
\hline & Si & No & \\
\hline & $\%(n)$ & $\%(n)$ & \\
\hline \multirow[t]{2}{*}{ VIH/SIDA } & $\sigma^{\top}$ & $22,7(5)$ & $27,2(6)$ \\
\hline & ? & $4,5(1)$ & 45,4 (10) \\
\hline \multirow[t]{2}{*}{ CANCER } & $\sigma^{\top}$ & $12,5 \%$ & $25(2)$ \\
\hline & q & - & $62,5(5)$ \\
\hline
\end{tabular}

Tabla 8. Uso de la Estrategia de Afrontamiento para Evitar Problemas

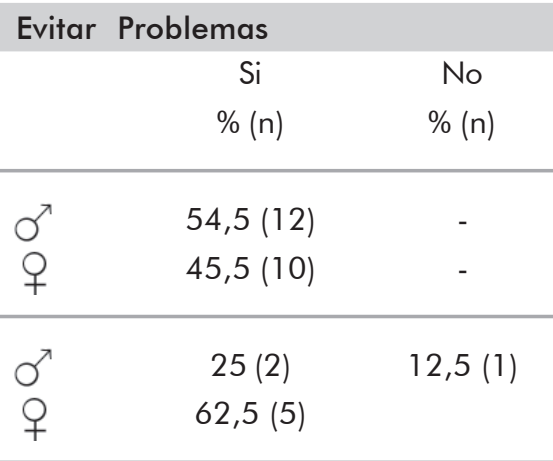

Tabla 9. Uso de la Estrategia de Afrontamiento Retirada Social

\begin{tabular}{|c|c|c|c|}
\hline \multicolumn{4}{|c|}{ Retirada Social } \\
\hline & & $\mathrm{Si}$ & No \\
\hline & & $\%(n)$ & $\%(n)$ \\
\hline \multirow[t]{2}{*}{ VIH/SIDA } & $\sigma^{\top}$ & $50(11)$ & $4,5(1)$ \\
\hline & q & $45,5(10)$ & - \\
\hline \multirow[t]{2}{*}{ CANCER } & $\sigma^{\top}$ & $37,5(3)$ & - \\
\hline & q & $62,5(5)$ & - \\
\hline
\end{tabular}

Tabla 10. Uso de las Estrategias de afrontamiento en relación con la edad y diagnóstico.

\begin{tabular}{|c|c|c|c|c|c|}
\hline \multicolumn{6}{|c|}{ Afrontamiento } \\
\hline & & & Escaso UE & Adecuado UE & Total \\
\hline \multirow[t]{6}{*}{$\mathrm{VIH}$} & EDAD & $20-30$ & 2 & 0 & 2 \\
\hline & & $31-40$ & 11 & 1 & 12 \\
\hline & & $41-50$ & 2 & 0 & 2 \\
\hline & & $51-60$ & 1 & 0 & 1 \\
\hline & & $61-70$ & 1 & 4 & 5 \\
\hline & & Total & 17 & 5 & 22 \\
\hline \multirow[t]{5}{*}{ CANCER } & EDAD & $31-40$ & 1 & 0 & 1 \\
\hline & & $41-50$ & 1 & 0 & 1 \\
\hline & & $61-70$ & 0 & 1 & 1 \\
\hline & & $71-80$ & 5 & 0 & 5 \\
\hline & & Total & 7 & 1 & 8 \\
\hline
\end{tabular}

$* U E=$ Uso de estrategias 


\section{Discusión}

Con base a lo realizado dentro de una clínica privada de la ciudad de Santa Marta, se trabajó en el área de hospitalización, esta fue una iniciativa apoyada por los diferentes profesionales de la salud que laboran dentro de la institución y sustentada teóricamente para hacer más valida su aplicabilidad; se contó a su vez con el recurso humano e instrumental para llevar a cabo el procedimiento evaluativo, con el objetivo de identificar y analizar en los pacientes con VIH y Cáncer el uso adecuado de estrategias de afrontamiento, con la finalidad de facilitarles un mejor proceso de adaptación a la enfermedad y lograr una modificación de esquemas y comportamientos en relación a su salud.

De manera general se encontró que un $80 \%$ de la población valorada no cuenta con la capacidad intelectual y emocional para afrontar la situación adversa que presenta (Tabla 11). Al evidenciarse un escaso uso en estrategias como resolución de problemas, pensamiento desiderativo, alta autocritica, restructuración cognitiva, apoyo social, evitación del problema y retirada social. Dichos resultados muestran un contraste con el estudio de los investigadores $^{[25]}$, donde las estrategias de afrontamiento más utilizadas por la población evaluada en dicho estudio son las de aproximación. En estos pacientes predominan el optimismo, la esperanza y la búsqueda de apoyo para enfrentar la enfermedad.

En la estrategia de expresión emocional, se evidencia que el $57 \%$ de la muestra participante hace un uso de la misma, lo que establece una relación con el estudio realizado por Baiocchi y cols, ${ }^{[26]}$, quienes encontraron que entre el $56 \%$ y el $79 \%$ de los evaluados se enfocan en liberar la emoción, en el momento de sentir mucho malestar emocional, terminan por expresarlo al conversar con alguien más o reducir el estrés mediante el llanto.

Los resultados previos mostraron ${ }^{[27]}$ que los pacientes tenían una buena red de apoyo especialmente en la parte religiosa, con los profesionales de la salud, al igual que manejaban un adecuado control de emociones y muy baja reacción de agresividad, en comparación con el estudio realizado, los pacientes atendidos en la clínica privada de la ciudad de Santa Marta, de la población total, el 76\% (23 de los 30 pacientes evaluados) presentaron puntuaciones bajas en la variable de apoyo social, es decir que no presentan una red de soporte que les sirva como apoyo emocional.

Así mismo, no se estableció diferencia alguna entre el género, es decir que tanto hombres como mujeres presentan las mismas características en relación a los recursos de afrontamiento que asumen. En otra pesquisa se aprecia que los hombres adoptan un mecanismo de negación de su realidad con más frecuencia ${ }^{[13]}$. En este estudio, por su parte, el pensamiento desiderativo es frecuente por igual número tanto en hombres como mujeres.

Por otro lado, en esta investigación ${ }^{[18]}$ los pacientes evaluados presentaron situaciones donde emergen cogniciones fatalistas, negativas y de desesperanza, asociadas a la presencia de estados de-

\section{Tabla 11. Uso de las Estrategias de afrontamiento.}

\begin{tabular}{lc} 
Escaso UE & Adecuado UE \\
Porcentaje $(n)$ & Porcentaje $(n)$ \\
$20(24)$ & $20(6)$ \\
\hline
\end{tabular}

$* \mathrm{UE}=$ Uso de estrategias presivos con angustia y ansiedad. En la mayoría de estos casos se observó poca presencia de su grupo de apoyo primario y social. En el presente estudio de igual forma se hace un registro de que el $77 \%$ no cuenta tampoco con una red de apoyo familiar y/o social.

En estrategias como Resolución de Problemas el 100\% de la población evaluada no cuenta con un adecuado uso de esa estrategia; en lo que compete a las habilidades de afrontamiento como la Autocrítica, Retirada Social y Evitación del Problema solo un 10\% de los pacientes cuenta con la adecuada utilización de estos recursos, describiéndose como personas con baja capacidad de solucionar la situación, grados contradictorios entre muy bajos o altos de sentimiento de culpa, aislamiento e intentos de apartarse del problema, lo cual es coherente con lo hallado en las investigaciones realizadas $^{[11,18]}$, en la cuales se asocian a las personas diagnosticadas con VIH-Sida y CA sentimientos de culpa, baja autoestima e ideación suicida, a su vez suelen presentar aislamiento en las esferas laborales, sociales, familiares, al igual que sienten temor a ser blanco de discriminación y estigma social, poco control de emociones, en especial la ira, al igual que actitudes de conducta de resignación e inhibición de emociones y presentaban explosiones afectivas

\section{Conclusión}

Teniendo en cuenta los resultados evidenciados en la primera fase de la investigación, es decir la diagnóstica, se ve preciso mencionar que se establezca un programa de entrenamiento para el uso adecuado de estrategias de afrontamiento, esto en apoyo con las diversas áreas de intervención en las que se divide la clínica. Así mismo se propone que se organicen actividades que permitan al usuario y sus familiares, una mejor atención a sus interrogantes con relación a su cuadro clínico o procedimientos quirúrgicos de naturaleza ya sea ambulatoria u hospitalaria, capacitando un refuerzo del proceso psicoeducativo que se realiza con base a: Manejo de diagnóstico, adherencia al tratamiento, manejo de emociones negativas (ansiedad, tristeza, estrés, ira...) intervención en crisis, por mencionar solo algunas.

En áreas tales como Cirugía, Pediatría, Hemodinamia, Quimioterapia, UCI, UCI Neonatal, Tocófano, Observación y Urgencias, ya se ha venido trabajando en dichas necesidades desde hace años con intervención continua del departamento de Psicología Clínica, pero se hace imperante el refuerzo a estos ámbitos de trabajo, y que a largo plazo pueda reconocerse y/o mantenerse esta misma área para mejorar en atención al usuario dentro de la institución.

Dentro de los espacios para pacientes que pertenezcan a las áreas de Cirugía, Hemodinámica y Quimioterapia, se recomiendan actividades que estimulen la participación grupal, puesto que al intercambiar experiencias se lograría minimizar el estrés psicológico que caracteriza el someterse a intervenciones invasivas. A su vez se sugiere que ambienten las zonas donde el psicólogo realiza las actividades para que el manejo del paciente pueda ser adecuado y no haya interferencias dentro del proceso terapéutico.

El trabajo realizado se enmarca como un proyecto que se espera sea continuado, puesto que al presentar esta evidencia práctica se hace exclusiva la continuidad y el trabajo casi intensivo no solo con esta población vulnerable, sino aquellos que requieran de la intervención y estimulación en estilos de afrontamiento, buscando facilitarles una mayor comprensión del problema que asumen y a su vez, brindarles los primeros auxilios psicológicos sobre como confrontar aquellas situaciones problemas que se asocian a su enfermedad e interfieren con el proceso de bienestar en torno a su salud física y mental. 
Por último, cabe mencionar que como indicador de impacto se busca que aquellos pacientes que presentan una condición clínica angustiante logren mediantes sus propios recursos establecer un régimen de apoyo psicológico-emocional-social, y permitan de tal forma un mejor afrontamiento de su situación actual.

\section{Bibliografia}

1. Sánchez-Sosa, J.J., et al., Un modelo psicológico en los comportamientos de adhesión terapéutica en personas con VIH. Salud mental, 2009. 32(5): p. 389-397.

2. Gallo, R.C., Virus hunting: AIDS, cancer, and the human retrovirus: a story of scientific discovery. 1991: Basic Books.

3. Fahey, J.L., et al., The prognostic value of cellular and serologic markers in infection with human immunodeficiency virus type 1. N Engl J Med, 1990. 322(3): p. 166-72.

4. Godoy, J.F., Psicología de la salud: delimitación conceptual, in Manual de psicología de la salud. Fundamentos, metodología y aplicaciones, M.A. Simón, Editor. 1999: Madrid. p. 39-76.

5. Pascual, L.M. and R. Ballester, La práctica de la psicología de la salud: programas de intervención. Promolibro ed. 1997, Valencia.

6. Tobón, S. and A. Nuñez, Psicología y sistema de salud en Latinoamérica. Perspectivas en psicología, 2000. 3: p. 21-40.

7. Bayés, R., Psicología y SIDA: Análisis funcional de los comportamientos de riesgo y prevención. Papeles del Colegio de Psicólogos. 1990.

8. Bayes, R., SIDA y psicología. 1995: Martínez Roca.

9. Bayés, R. and P. Arranz, Las variables psicológicas como cofactores del síndrome de inmunodeficiencia adquirida. Jano, Medicinay Humanidades, 1998. 34(9): p. 1313.

10. Cahn, P., Abordaje Psicologico de Personas con VIH. 2013, Fundacion Huesped.

11. Ramirez Gonzales, V., Intervención psicológica en VIH/ SIDA.

12. Mendoza, J.E.V., M.C. Ramírez, and J.E.A. Morales, Estrategias de afrontamiento del sida en pacientes diagnosticados como seropositivos. Psicología y Salud, 2013. 19(2).

13. Gallar, M., Promoción de la Salud y Apoyo Psicológico al Paciente. 2006, Madrid: Thomson Learning Paraninfo.
14. Andreu, Y. Galdón. MJ (1989). Un primer acercamiento a la evaluación del ajuste emocional del paciente oncológico. in Anales de Psiquiatría.

15. Rowland, J.H., Developmental stage and adaptation: adult model. Handbook of psychooncology, 1989: p. 25-45.

16. Guadalupe, L.A.O., Manual de Psicologi Clinica y de la Salud Hospitalaria, ed. P. Editores. 2005, Bogota.

17. Montoya, D.N. and B.B. Vergara, Una mirada al impacto, reacciones emocionales y estilos de afrontamiento de los pacientes con cáncer.

18. Solana, C.A., Aspectos psicológicos en el paciente superviviente. Oncología, 2005. 28(3): p. 151-163.

19. Lazarus, R.S. and S. Folkman, Estrés y procesos cognitivos, ed. S.P. Company. 1984, New York.

20. Forsythe, C. and B. Compas, Interaction of cognitive appraisals of stressful events and coping: Testing the goodness of fit hypothesis. Cognitive Therapy and Research, 1987. 11 (4): p. 473-485.

21. Mejorada, R.E.O., et al., Afrontamiento en pacientes con cáncer de mama en radioterapia: análisis de la Escala COPE Breve. Psicología y Salud, 2013. 23(1).

22. Barros Sánchez, S., et al., Capacidad de afrontamiento y adaptación de pacientes con VIH-SIDA según modelo "Callista Roy" en una IPS de II nivel, Cartagena 20122013. 2013.

23. Rivero Burón, R., et al., Psicología y cáncer. 2008, 2008. 15(1).

24. Martínez, J.G., Adaptación española del Inventario de Estrategias de Afrontamiento. Actas Esp Psiquiatr, 2007. 35(1): p. 29-39.

25. Coppari, N., et al., Calidad de vida y afrontamiento de pacientes con cáncer y sida de Paraguay//Quality of life and coping of cancer and aids patients in Paraguay. Salud \& Sociedad, 2014. 5(1).

26. Antonet, B.-M., et al., Estilos de afrontamiento en pacientes con cáncer de cuello uterino. Servicio de ginecología de un instituto nacional.

27. Gaviria, A.M., et al., Quality of life related to health, coping with stress and negative emotions in cancer patients in chemotherapy treatment. Psicología desde el Caribe, 2007(20): p. 26. 